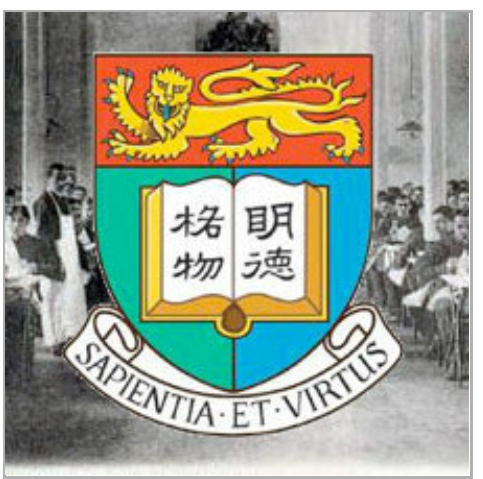

SEP 15, 2019

\section{open ठAccess}

\section{DOI:}

dx.doi.org/10.17504/protocol s.io.6yahfse

\section{Protocol Citation: Scott C} Edmunds, Jesse Xiao, Hongling Zhou 2019. University Published Paper Reproducibility Assessment Protocol - For students. protocols.io

https://dx.doi.org/10.17504/p rotocols.io.6yahfse

License: This is an open access protocol distributed under the terms of the Creative Commons Attribution License, which permits unrestricted use, distribution, and reproduction in any medium, provided the original author and source are credited

Protocol status: Working We use this protocol and it's working

Created: Aug 30, 2019

Last Modified: Sep 15, 2019

PROTOCOL integer ID: 27362

\section{(3) University Published Paper Reproducibility Assessment Protocol - For students}

In 1 collection

\author{
Scott C \\ Edmunds $^{1}$, \\ Jesse $\mathrm{Xiao}^{2}$, Zhou ${ }^{3}$ \\ ${ }^{1}$ GigaScience/BGI Hong Kong/Bauhinia Genome; \\ ${ }^{2}$ GigaScience/BGI Hong Kong; ${ }^{3}$ GigaScience/BGI
}

\section{BGI}

Hongling Zhou

\section{ABSTRACT}

This protocol is for students carrying out a literature/data curation exercise to crowdsource and determining whether the data availability of papers from a university meets the national, funder, university and journal policies. The teachers setting up this assessment have a separate related protocol they need to follow, and they will ultimately collate and mark these results. 
Keywords: Open Data,

Reproducibility, Reproducible

Research

\section{Find the assigned papers}

Each student should have been assigned a number of papers in a spreadsheet that they need to assess.

1.1 Find your assignments, and start the exercise by looking at the first assigned paper, clicking on the identifier (handle, URL, etc.) and locating the paper to assess via its DOI.

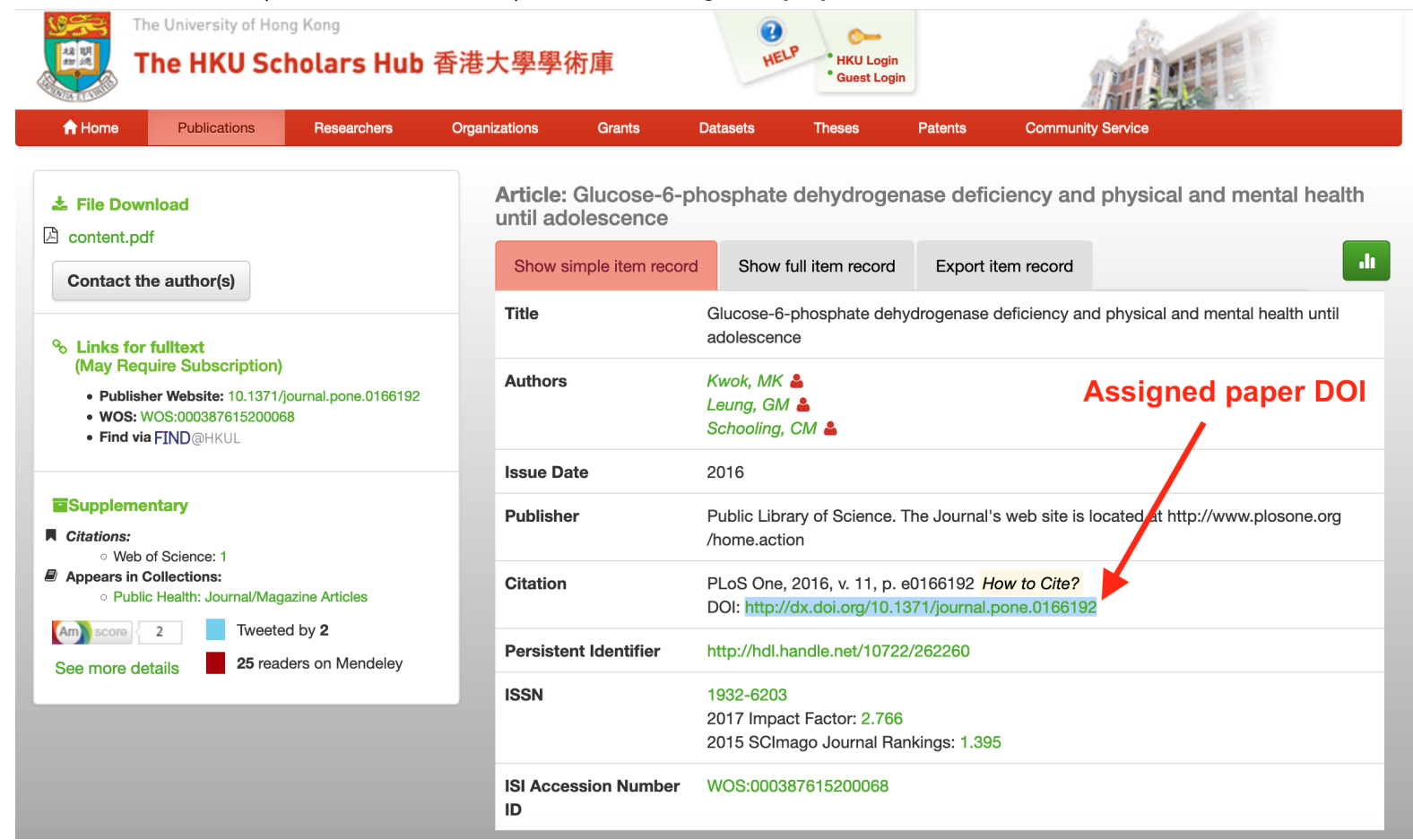

University IR/CRIS landing page showing the assigned paper DOI that needs to be clicked on and assessed.

\section{Assess the assigned paper to see if there is data}

Quickly scan through the first paper on the list alongside any supplemental files to look for mentions of any supporting data. If there is no data in the paper (e.g. it is an opinion article, an erratum, covers a purely theoretical subject, or some other type of paper purely discussing rather than carrying out data production) then mark it as not having data and go to the next paper on the list. 


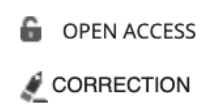

\title{
Correction: EZH2-Mediated H3K27me3 Is Involved in Epigenetic Repression of Deleted in Liver Cancer 1 in Human Cancers
}

Sandy Leung-Kuen Au, Carmen Chak-Lui Wong, Joyce Man-Fong Lee, Chun-Ming Wong, Irene Oi-Lin Ng

Published: January 24, 2014 • https://doi.org/10.1371/annotation/9085e9bd-25b1-4266-b6db-34e56d80ce3a

\begin{tabular}{|l|l|l|l|}
\hline Article & Metrics & Comments & \\
$\qquad$ & & \\
\hline
\end{tabular}

Reader Comments (0)

Media Coverage

\begin{abstract}
An error occurred which caused only one of the two designated corresponding authors to appear. Dr. Chun-Ming Wong is also a corresponding author and can be contacted at jackwong@pathology.hku.hk.
\end{abstract}

Citation: Au SL-K, Wong CC-L, Lee JM-F, Wong C-M, Ng IO-L (2014) Correction: EZH2-Mediated H3K27me3 Is Involved in Epigenetic Repression of Deleted in Liver Cancer 1 in Human Cancers. PLoS ONE 9(1): 10.1371/annotation/9085e9bd25b1-4266-b6db-34e56d80ce3a. https://doi.org/10.1371/annotation/9085e9bd25b1-4266-b6db-34e56d80ce3a

Published: January 24, 2014

Copyright: (c) 2014 . This is an open-access article distributed under the terms of the Creative Commons Attribution License, which permits unrestricted use, distribution, and reproduction in any medium, provided the original author and source are credited.

Competing interests: No competing interests declared.

Example of a Correction/Errata article that does not present any data. This should be marked as not having any data and the student should move on to the next paper.

\section{If there is data, find it in the paper}

3.1 You will not need to properly read and digest the contents of the paper, but aim to spend 5-10 
minutes going through the manuscript, trying to understand what type of experiments have been carried out, and what likely data has been produced in the process of publishing the research.

3.2 In particular, concentration should be given for checking the Methods, Results and if there is a specific "Data Availability" section in the paper. PLOS for example has a "Data Availability" section in their structured abstract/article front page and a "Supporting information" section at the end of their papers.

\section{PLOS | ONE}

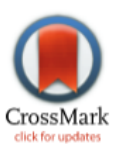

\section{G OPENACCESS}

Citation: Kwok MK, Leung GM, Schooling CM (2016) Glucose-6-Phosphate Dehydrogenase Deficiency and Physical and Mental Health until Adolescence. PLOS ONE 11(11): e0166192. doi:10.1371/journal.pone. 0166192

Editor: Junwen Wang, Mayo Clinic Arizona, UNITED STATES

Received: August 10, 2016

Accepted: October 24, 2016

Published: November 8, 2016

Copyright: $\odot 2016$ Kwok et al. This is an open access article distributed under the terms of the Creative Commons Attribution License, which permits unrestricted use, distribution, and reproduction in any medium, provided the original author and source are credited.

Data Availability Statement: Data are available upon request from the "Children of 1997 ' data access committee: aprmay97@hku.hk. The volume and complexity of the data collected precludes public data deposition, because the participants could be identifiable from such extensive data which would comprise participant privacy.

Funding: This work was a sub-study of the "Children of 1997" birth cohort which was initially supported by the Health Care and Promotion Fund, Health and Welfare Bureau, Government of the Hong Kong SAR [HCPF Grant \# 216106] and reestablished in 2005 funded by the Health and

\section{RESEARCH ARTICLE}

\section{Glucose-6-Phosphate Dehydrogenase Deficiency and Physical and Mental Health until Adolescence}

\author{
Man Ki Kwok', Gabriel M. Leung ${ }^{1}$, C. Mary Schooling ${ }^{1,2 *}$
}

1 School of Public Health, Li Ka Shing Faculty of Medicine, The University of Hong Kong, Hong Kong Special Administrative Region, China, 2 City University of New York Graduate School of Public Health and Health Policy, New York, New York, United States of America

* cms1@ @ku.hk

\section{Abstract}

\section{Background}

To examine the association of glucose-6-phosphate dehydrogenase (G6PD) deficiency with adolescent physical and mental health, as effects of G6PD deficiency on health are rarely reported.

\section{Methods}

In a population-representative Chinese birth cohort: "Children of 1997" ( $n=8,327)$, we estimated the adjusted associations of G6PD deficiency with growth using generalized estimating equations, with pubertal onset using interval censored regression, with hospitalization using Cox proportional hazards regression and with size, blood pressure, pubertal maturation and mental health using linear regression with multiple imputation and inverse probability weighting.

Results Structured data

\section{Results availability section}

Amo yro,520 screened adolescents ( $66 \%$ follow-up), $4.8 \%$ boys and $0.5 \%$ girls had G6PD deficiency. G6PD-deficiency was not associated with birth weight-for-gestational age or length/height gain into adolescence, but was associated with lower childhood body mass index (BMI) gain ( $-0.38 \mathrm{z}$-score, $95 \%$ confidence interval $(\mathrm{Cl})-0.57,-0.20)$, adjusted for sex and parental education, and later onset of pubic hair development (time ratio $=1.029,95 \%$ $\mathrm{Cl} 1.007,1.050)$. G6PD deficiency was not associated with blood pressure, height, BMI or mental health in adolescence, nor with serious infectious morbidity until adolescence.

\section{Conclusions}

G6PD deficient adolescents had broadly similar physical and mental health indicators, but transiently lower BMI gain and later pubic hair development, whose long-term implications warrant investigation. 
3.3 Make a note if all of the data is in the manuscript itself (figures, tables or included supplemental files) or accessible via external sources - e.g. via database accession numbers or linked via URLs or data DOIs to data repositories such as figshare, zenodo or Dryad.

3.4 If data is only available on request make a note of the reasons given and details of who to contact to access the data. Having access to the raw data is most important, so make a note in the comments section of the spreadsheet if that is available.

\section{$4 \quad$ Check external sources for data}

If external data sources have been mentioned, e.g. database accessions or URL/DOI links to datasets, check if these are working by clicking on the URLs or finding the online databases mentioned.

4.1 If a database and accession number is listed, access the database mentioned in the paper and search it for the accession number (input the accession number and click search).

NIH U.S. National Library of Medicine

Search NCBI

MK033747

Search

Search results for: MK033747

\section{Results by database}

Results found in 0 databases

\begin{tabular}{|c|c|c|c|c|c|}
\hline \multicolumn{2}{|l|}{ Literature } & \multicolumn{2}{|l|}{ Genes } & \multicolumn{2}{|l|}{ Proteins } \\
\hline Bookshelf & 0 & Gene & 0 & Conserved Domains & 0 \\
\hline MeSH & 0 & GEO DataSets & 0 & Identical Protein Groups & 0 \\
\hline NLM Catalog & 0 & GEO Profiles & 0 & Protein & 0 \\
\hline PubMed & 0 & HomoloGene & 0 & Protein Clusters & 0 \\
\hline \multirow[t]{2}{*}{ PubMed Central } & 0 & PopSet & 0 & Sparcle & 0 \\
\hline & & & & Structure & 0 \\
\hline
\end{tabular}

Example of an external data source: checking the NCBI database (www.ncbi.nlm.nih.gov) to see if the data accession number given in the paper is working. 
4.2 If a URL or DOI is listed in the paper as being the source of the data, test to see if it works.

\section{Note}

In both cases test if the datasets are available for download and there is not just a landing page

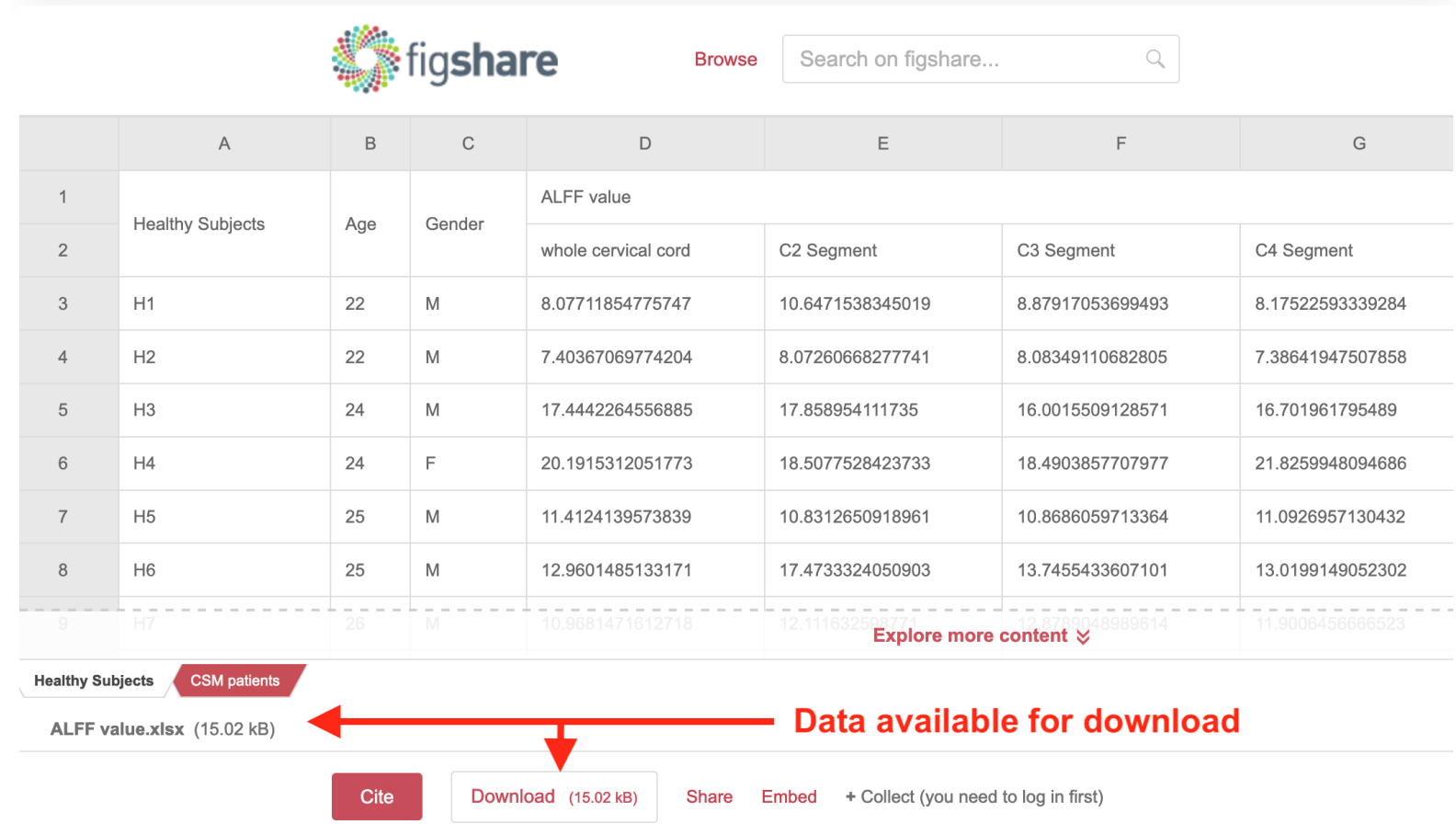

Example of a figshare dataset entry linked via DOI from a paper where the data (.xIsx file) is available for download. Source: http://dx.doi.org/10.6084/m9.figshare.4233887

\section{Summarise results on whether data is available}

Once you have checked the paper and any supplemental and external data sources for data and input all of this information into the spreadsheet, make an overall rating on whether you feel the data is available without restriction, if data is available on request, or if data is missing. Once this example is complete go to the next paper on the list and assess that in the same manner. Once this literature curation exercise is complete send the results back to the teacher to be marked and collated alongside all of the other crowdsourced results from the other students. 